\title{
High-throughput sequencing identifies aetiology-dependent differences in ductular reaction in human chronic liver disease
}

Olivier Govaere ${ }^{1,2^{*}}$, Simon Cockell ${ }^{3}$, Matthias Van Haele ${ }^{1}$, Jasper Wouters ${ }^{4,5}$, Wouter Van Delm ${ }^{6}$, Kathleen Van den Eynde ${ }^{1}$, Arianna Bianchi ${ }^{2}$, Rudy van Eijsden ${ }^{6}$, Werner Van Steenbergen ${ }^{7}$, Diethard Monbaliu ${ }^{8}$, Frederik Nevens $^{7}$, Tania Roskams $^{1}$

${ }^{1}$ Department of Imaging and Pathology, KU Leuven and University Hospitals Leuven, Leuven, Belgium

${ }^{2}$ Institute of Cellular Medicine, Newcastle University, Newcastle upon Tyne, United Kingdom

${ }^{3}$ Bioinformatics Support Unit, Newcastle University, Newcastle upon Tyne, United Kingdom

${ }^{4}$ VIB Center for Brain \& Disease Research, KU Leuven, Leuven, Belgium

${ }^{5}$ Department of Human Genetics, KU Leuven, Leuven, Belgium

${ }^{6}$ VIB Nucleomics Core, KU Leuven, Leuven, Belgium

${ }^{7}$ Department of Hepatology, KU Leuven and University Hospitals Leuven, Leuven, Belgium

This article has been accepted for publication and undergone full peer review but has not been through the copyediting, typesetting, pagination and proofreading process which may lead to differences between this version and the Version of Record. Please cite this article as doi: $10.1002 /$ path.5228 
${ }^{8}$ Department of Abdominal Transplant Surgery, KU Leuven and University Hospitals Leuven, Leuven, Belgium

${ }^{*}$ Correspondence to: Dr. Olivier Govaere, MSc, PhD, Institute of Cellular Medicine Room M4.115, Leech Building, Newcastle University Medical School, Framlington Place, Newcastle upon Tyne, NE2 4HH, UK. E-mail: olivier.govaere@ncl.ac.uk

Short running title: Aetiology-dependent differences in the human hepatic ductular reaction

Conflict of interest statement: The authors declare no conflict of interest.

\section{Word count and number of figures/tables}

3,664 words. 4 figures and 1 table.

\section{RNA sequencing GEO data}

GEO number: GSE118373

Access token: ohszyiomdtyjdil

Website: https://www.ncbi.nlm.nih.gov/geo/query/acc.cgi?acc=GSE118373 


\section{Abstract}

Ductular reaction (DR) represents the activation of hepatic progenitor cells (HPCs) and has been associated with features of advanced chronic liver disease; yet it is not clear whether these cells contribute to disease progression and how the composition of their micro-environment differs depending on the aetiology. This study aimed to identify HPC-associated signalling pathways relevant in different chronic liver diseases using a high-throughput sequencing approach. DR/HPCs were isolated using laser microdissection from patient samples diagnosed with hepatitis $C$ virus (HCV) or primary sclerosing cholangitis (PSC), as models for hepatocellular or biliary regeneration. Key signals were validated at the protein level for a cohort of 56 patients (20 early and 36 advanced stage). In total, 330 genes were significantly differentially expressed between the HPCs in HCV and PSC. Recruitment and homing of inflammatory cells were distinctly-different depending on the aetiology. HPCs in PSC were characterised by a response to oxidative stress (e.g. JUN, VNN1) and neutrophil-attractant chemokines (CXCL5, CXCL6, IL-8), whereas HPCs in HCV were identified by T- and B-lymphocyte infiltration. Moreover, we found that communication between HPCs and macrophages was aetiology driven. In PSC, a high frequency of CCL28-positive macrophages was observed in the portal infiltrate, already in early disease in the absence of advanced fibrosis, while in HCV, HPCs showed a strong expression of the macrophage scavenger receptor MARCO. Interestingly, DR/HPCs in PSC showed more deposition of extracellular matrix (e.g. FN1, LAMC2, collagens) compared to HCV, where an increase of pro-invasive 
genes (e.g. PDGFRA, IGF2) was observed. Additionally, endothelial cells in the vicinity of DR/HPCs showed differential immunopositivity (e.g. IGF2 and INHBA expression). In conclusion, our data shine light on the role of DR/HPCs in immune signalling, fibrogenesis and angiogenesis in chronic liver disease.

\section{Key words}

Regeneration, progenitor cell niche, tissue remodelling, immunity, fibrogenesis, differentiation 


\section{Introduction}

Chronic liver disease is associated worldwide with a high morbidity and mortality rate. About $0.1 \%$ of the European population is affected by cirrhosis, corresponding to 14 to 26 new cases per 100,000 inhabitants per year, or an estimated 170,000 deaths yearly [1]. Orthotopic liver transplantation is still the best curative option for end-stage liver disease although donor organ availability cannot meet the demand, meaning that many patients die whilst waiting for a suitable organ.

Persistent chronic injury of the liver epithelial cells leads to oxidative stress and senescence, inhibiting the capacity to proliferate, and is believed to be a major contributor in the progression of age-related liver diseases [2]. In response to this cell damage, a reserve epithelial cell compartment becomes active, the hepatic progenitor cells (HPCs), and attempts to restore liver homeostasis by differentiating into either cholangiocytes or hepatocytes depending on the underlying disease [2]. Activation of HPCs is usually seen as a ductular reaction (DR) which comprises an expansion of transit amplifying cells of the terminal branches of the biliary tree located in the Canal of Hering [3]. In both acute and chronic liver diseases the degree of DR has been reported to reflect disease severity and correlates with shortterm mortality [4-6].

HPCs reside in a specialised micro-environment, their so-called niche, which is crucial in determining their cell fate. Laminins, as part of the extracellular matrix (ECM), have been reported to control the expansion of HPCs in an undifferentiated 
state, and hence DR, during liver injury [7]. The reduction of laminin deposition by treatment with iloprost has been described to result in enhanced differentiation of HPCs into hepatocytes in a mouse model of chronic parenchymal damage [8]. The disruption of the integrin receptor $\beta 6$, an adhesion receptor that interacts with fibronectin and transforming growth factor beta 1, inhibits the response of HPCs to tissue damage [9].

Regeneration and injury are often described separately as discrete events, however the inflammatory response does not only play a role in fibrosis but is also crucial for liver regeneration. $[10,11]$. Macrophages in particular have been described to influence the polarisation and invasion of HPCs into damaged parenchyma [12]. Additionally they are a source of Wnt3a that stimulates hepatocyte differentiation in rodents [13]. HPCs have a typical biliary phenotype meaning they express cholangiocyte markers, such as keratin (K) 19 and EPCAM, which they gradually lose upon differentiation towards hepatocytes [2]. Several pathways have been described to drive HPCs towards a specific cell fate. The Notch and YAP-1 pathway are important for cholangiocyte differentiation while inhibition of those pathways commits HPCs to hepatocytes $[13,14]$.

DR has been associated with fibrosis and portal inflammation in chronic liver diseases though the differences between aetiologies are still poorly understood [4, 15-17]. A thorough identification of the hepatic DR and of the signals that govern the proliferation, neo-angiogenesis and differentiation of HPCs into mature epithelial 
cells might lead to the development of clinically feasible methods to induce liver repopulation from these endogenous cells. This study aims to characterise the human hepatic DR/HPCs and its surrounding in chronic liver disease by using a high-throughput sequencing approach. Samples obtained from patients diagnosed with primary sclerosing cholangitis (PSC) or hepatitis $\mathrm{C}$ virus (HCV) were included in this study as examples of pure biliary or hepatocellular damage and regeneration.

\section{Materials and methods}

\section{Patient selection}

Sixty seven patients diagnosed with primary HCV or PSC without any secondary aetiology and treated at the University Hospitals Leuven, Belgium between 2008 and 2014 were included in this study. Diagnosis was based on the WHO criteria. Patients with recurrent $\mathrm{HCV}$ infection or features of PSC following a liver transplantation were excluded. The explorative cohort consisted of eleven snap frozen samples obtained from end-stage diseased explant livers (PSC n=6, HCV n=5). Clinical data can be found in supplementary material, Table S1. The validation cohort comprised 56 formalin-fixed paraffin-embedded (FFPE) liver samples: 36 advanced disease (F3-F4 fibrosis, $n=18$ per group) and 20 diagnostic needle biopsies (F0-F1 fibrosis, $n=10$ per group). This retrospective study was approved by the ethical committee of the University Hospitals Leuven, Belgium. 
Laser capture microdissection

Cryosections cut at $10 \mu \mathrm{m}$ thickness were mounted onto metal frame polyethylene terephthalate slides (Leica Microsystems Gmbh, Wetzlar, Germany). DR/HPCs were visualised on the first slide by immunohistochemistry using a mouse anti-human keratin 7 (RTU, Dako, Glostrup, Denmark) as described below and the cell populations of interest were isolated on consecutive slides. These slides were fixed at room temperature in a $95 \%$ ethanol solution for $1 \mathrm{~min}$ and subsequently transferred to $75 \%$ and $50 \%$ ethanol for 15 s before staining with a $2 \%$ Cresyl Violet solution for 2 min (Ambion Inc., Foster City, CA, USA). The sections were dehydrated using an ethanol series (50\%, 75\%, 95\%, 100\%). Laser Capture Microdissection was performed on the Leica LMD 6500 system (Leica Microsystems). An average area of $10^{6} \mu \mathrm{m}^{2}$ was isolated for each population. Total mRNA was extracted using the RNeasy ${ }^{\circledR}$ Plus Micro kit (Qiagen, Hilden, Germany) according to the manufacturer's instructions.

High-throughput RNA sequencing

RNA integrity (RIN) was assessed using the Agilent RNA 6000 Pico kit (Agilent, Santa Clara, CA, USA) and had an average value of $6.61 \pm 0.47$ (SD). The SMARTer Universal Low Input RNA Kit (Clontech Laboratories, Mountain View, CA, USA) was used to generate cDNA. Samples were fragmented with the Adaptive 
Focused Acoustics $^{\text {TM }}$ (AFA) shearing method (Covaris Inc., Woburn, MA, USA) and subsequently processed with the NEBNext Ultra Library Prep kit (New England Biolabs, Ipswich, MA, USA). Sequencing was performed on the HiSeq 2000 platform (Illumina Inc., San Diego, CA, USA). Data are available on the NCBI GEO repository (GSE118373).

\section{Bioinformatics}

Reads that were shorter than $35 \mathrm{bp}$ after adapter trimming with FastX 0.0 .13 were removed. RNA sequencing reads were aligned to the reference genome GRCh37.73 using Tophat v2.0.8b and quality filtered with SAM Tools v0.1.19 [18, 19]. Counting was performed with htseq-count 0.5.4p3 [20]. Statistical comparative analysis was undertaken in R/Bioconductor using edgeR 3.4.0 to fit a genewise negative binomial generalised linear model [21]. Genes were considered differentially expressed if they exhibited an absolute fold change $>1$ and a p-value $<0.001$. Data were further analysed using STRING, Gene Set Enrichment Analysis and Ingenuity® Pathway Analysis (Qiagen), and visualised using the Cytoscape plugin EnrichmentMap 3.0 and GOPlot 1.0.2 [22-26].

Immunohistochemistry

FFPE and frozen human samples were stained using the Bond ${ }^{\mathrm{TM}}$ Polymer Refine Detection kit on a Bond Max autostainer (Leica). Heat-induced epitope retrieval was performed using citrate or EDTA buffers. Primary antibodies were directed against C-C motif chemokine ligand 28 (CCL28; GTX108432, GeneTex, Irvine, CA, USA; 
citrate, 1/200), CD34 (QBEnd 10, Dako; EDTA, ready-to-use), collagen type XVII alpha 1 chain (COL17A1; HPA043673, Sigma-Aldrich, St. Louis, MO, USA; citrate, 1/200), fibronectin 1 (FN1; A0245, Dako; EDTA, ready-to-use), hepatocyte nuclear factor 4 alpha (HNF4A; HPA004712, Sigma-Aldrich; EDTA, 1/50), Inhibin Subunit Beta A (INHBA; HPA020031, Sigma-Aldrich; citrate, 1/50), Insulin Like Growth Factor 2 (IGF2; MAB2921, R\&D systems, Minneapolis, MN, USA; citrate, 1/500), Jun proto-oncogene, AP-1 transcription factor subunit (JUN/c-Jun; ab32137, Abcam, Cambridge, UK; EDTA, 1/200), keratin 7 (K7; OV-TL 12/30, Dako; EDTA, ready-touse), keratin 19 (K19; RCK108, Dako; EDTA, ready-to-use), laminin subunit gamma 2 (LAMC2; HPA024638, Sigma-Aldrich; citrate, 1/500), macrophage receptor with collagenous structure (MARCO; HPA008847, Sigma-Aldrich; citrate, 1/50) and Neural Cell Adhesion Molecule 1 (NCAM1; 123C3, Dako; EDTA, ready-to-use). Horseradish peroxidase visualisation was performed with 3,3'-diaminobenzidine or 3amino-9-ethylcarbazole (Leica). Haematoxylin was used as a counterstain (Leica). Picro-Sirius Red staining was using a Leica ST5010 Autostainer and sections subsequently processed for IHC on a Bond Max (Leica). Quantification of immunopositive cells was done in three high power fields of the portal tract area in the end-stage specimens and at least one in the needle biopsies (magnification 400X). An absolute quantification was used for CCL28 and for MARCO an intensity score range $(0=$ negative, $1=$ weak, $2=$ moderate, $3=$ strong $)$. CD34 positivity was analysed with Image J based on staining without any haematoxylin counterstaining (https://imagej.nih.gov/ii). The statistical significance of differences was evaluated 
using an unpaired Student's $t$-test. Significant differences between experimental groups were ${ }^{*} p<0.05,{ }^{* *} p<0.01,{ }^{* *} p<0.001$.

\section{Results}

High-throughput RNA sequencing stratifies DR from parenchyma

DR in the periportal area, also referred to as Type 2A DR, and its close surrounding area with pericellular fibrosis were isolated from frozen end-stage liver samples with underlying $\mathrm{HCV}(n=5)$ and PSC $(n=6)$ [3]. Additionally, matching parenchyma were isolated from six patient samples, three per aetiology. A keratin 7 immunostain was used to discern the HPCs from intermediate or dedifferentiated hepatocytes (Figure 1A). DR in PSC showed the typical ductal plate formation at the interphase between the portal tract and parenchyma (Figure 1A). Heatmap plots of RNA-seq data revealed a distinct separate clustering of the hepatocytes and the DR. When comparing DR with hepatocytes, 2451 genes were differentially expressed in HCV and 1696 in PSC (Figure 1B). Both diseases had 512 up-regulated genes in common in the isolated DR/HPC population when compared to the corresponding hepatocytes, including genes as GSTP1, FZD1, CD44, JAG1, S100A6 and ANXA4 (supplementary material, Table S2). Gene set enrichment analysis showed an increase in networks involving ECM, adhesion and immune signalling in the DR of both diseases, while nodes up-regulated in the hepatocytes clustered in metabolic networks (e.g. lipid metabolism, oxidation-reduction reactions, carbohydrate 
metabolism and catabolic processes) (Figure 1C,D). Additionally, the DR in PSC showed networks enriched in morphogenesis and cytoskeleton organisation, whereas in HCV enrichment for cell movement and receptor activity was observed. Taken together these results show that DR/HPCs have a distinct different gene signature from the liver parenchyma in both aetiologies.

DR is associated with aetiology-dependent inflammatory signatures and transcriptional regulators

To investigate the differences in DR between the different aetiologies, we performed a direct comparison of the DR transcriptome from $\mathrm{HCV}(n=5)$ and PSC $(n=6)$. In total 330 genes were differentially expressed between the two aetiologies, 141 associated with HCV and 189 with PSC (supplementary material, Table S3). The top 40 differentially expressed genes based on $\mathrm{P}$ value are listed in Table 1. Gene Ontology clustering of the 330 differentially expressed genes revealed an enrichment in genes related to 'regulation of immune system process', 'extracellular region', 'regulation of lymphocyte and leucocyte activation', 'regulation of cell activation' and 'regulation of response to stimulus' (Figure 2A and supplementary material, Figure S1).

Furthermore, DR from PSC samples showed an increase in the HPC/biliary markers EPCAM, TACSTD2, PROM1 and NCAM1, whereas DR from HCV only showed an increase in some metabolism-/hepatocyte-markers (e.g. CYP1A1 and FADS2) (supplementary material, Table S3). Of note, no significant differences were found in 
the expression of $K R T 19, K R T 7$ or $S O X 9$, suggesting that a similar number HPCs was isolated in both aetiologies. Histological analysis showed that HCV samples were characterised by lymphoid aggregates at the peri-portal area, while PSC samples showed a high infiltration of neutrophils, typical features used in the clinicopathological diagnosis of the diseases, confirming the observed gene signatures (Figure 2B). Immunohistochemical analysis for NCAM1 showed an abundant expression in the HPCs of PSC when compared to HCV, which is consistent with the gene expression (Figure 2B).

Using the Regulator Effect tool of Ingenuity Pathway Analysis (with a cut-off of absolute activation Z-score $>2$ ) several transcriptional regulators were identified in HCV (SMAD7, MYCN, HNF4A, KLF2 and PAX5) and PSC (NFKBIA, REL, STAT3, CTNNB1, CDKN2A, JUN, TP63, KLF4, NFE2L2 and STAT4) (Figure 2C). Nuclear HNF4A was observed focally in the HCV-associated DR/HPCs, in contrast nuclear JUN expression was more predominant in PSC compared to HCV (Figure 2 D,E). Interestingly, a weak and focal HNF4A positivity was observed in the intermediate ductules and bile ducts of HCV samples, while the bile ducts in PSC samples showed a strong nuclear JUN expression compared to HCV (supplementary material, Figure S2). Taken together, analysis of all the differentially expressed genes indicates aetiology-dependent differences and activation of specific transcriptional regulators. 
Dynamics in immune signalling associated with DR

In order to better understand the differences in DR, especially regarding to inflammatory signals, the genes associated with HCV $(n=141)$ and the genes associated with PSC $(n=189)$ were compared with each other. Applying STRING analysis to predict protein-protein interactions indicated an up-regulation of the GOnetwork 'Positive regulation of immune system response" (FDR<0.001) in the HPCs of HCV, and an up-regulation of "ECM organisation" (FDR $<0.001)$ and "Cytokine activity" in PSC (supplementary material, Figure S1). Ingenuity Pathway Analysis showed a similar shift in 'Diseases and Biological Functions' in both aetiologies, e.g. 'cell movement', 'inflammatory response' and 'growth of connective tissue' (supplementary material, Figure S3). Although DR in both diseases showed enriched GO annotations for 'immune response process', 'extracellular space' and 'cell adhesion', differences were observed in genes associated with these pathways (Figure 3A). DR in HCV showed an enriched expression of CD5L, CD19, CD22, $H L A-D Q A 2$ and the macrophage scavenger receptor MARCO, while PSC-associated DR had high expression of neutrophil-chemoattractant genes CXCL1, CXCL6, CXCL5 and CXCL8, and cytokines CCL28, INHBA. Moreover, the HPC niche in PSC displayed in an increase in fibronectin 1 (FN1), LAMC2 and collagens (COL1A1, COL3A1, COL4A1, COL9A2 and COL10A1), while in HCV an increase of invasionrelated markers was observed (e.g. PDGFRA, IGF2) (Figure 3A). Histopathological analysis showed a 2.6-fold increase of infiltrating CCL28-positive macrophages in the portal area of PSC-related end-stage cirrhotic liver samples compared to HCV 
$(p<0.001)$ (Figure 3B). No epithelial positivity was observed for CCL28. Interestingly, the infiltrating CCL28-macrophages were more prominent in areas with DR than in areas with larger bile ducts (supplementary material, Figure S2). In early stage disease, fibrosis stage F0 to F1, a 3.7-fold increase of CCL28-positive cells was seen in PSC when compared to HCV $(p<0.01)$ (Figure 3B). Immunopositivity for MARCO was observed in the Kuppfer cells of all liver samples and focally a membranous positivity was seen in the HPCs. Interestingly, the HPCs in cirrhotic HCV samples showed a stronger intensity in MARCO expression, a 1.3-fold increase $(p<0.01)$, when compared to PSC samples (Figure $3 C$ and supplementary material, Figure S2). Moreover, focal positivity was observed in cholangiocytes of larger bile ducts in the end-stage HCV cohort (supplementary material, Figure S2). No significant differences in immunopositivity for MARCO were seen in early-stage liver diseases. These results demonstrate the aetiology-dependent dynamics of immune signalling in the HPC niche during chronic liver disease.

Spatial differences in ECM deposition and angiogenesis

Apart from the differences in up-stream regulators and immune signals in the DR, discrepancies in genes related to ECM deposition were observed. Overall, DR in PSC showed a strong increase in ECM-associated genes (e.g. COL1A1, COL3A1, COL9A2, COL10A1, LAMC2, FN1, FNDC1, STMN2), while DR in HCV only showed an increase in COL17A1 and the ECM degrading enzyme ADAMTS5. At the protein 
level, COL17A1 was observed in the cytoplasm of and in the basal membrane surrounding HPCs in end-stage HCV. Moreover, sinusoidal lining cells and a few cholangiocytes showed focal positivity for COL17A1 in HCV samples (supplementary material, Figure S2). Cytoplasmic LAMC2 positivity was mainly seen in HPCs of PSC samples (Figure 4A). The basal membrane surrounding the bile ducts and ductular reaction showed a weak positivity for LAMC2 in all the samples (Figure 4A and supplementary material, Figure S2). In addition, sequential staining showed that the HPCs in PSC were surrounded by dense FN1 deposition whereas areas of strong DR in HCV coincide with weak FN1 immunopositivity (Figure 4B). Furthermore, local differences within the same HCV samples were seen, especially between peri-portal areas, e.g. areas with $\mathrm{DR}$, and the central portal areas, e.g. areas with bile ducts (supplementary material, Figures S2 and S4). Areas with DR showed a 1.35 fold increase $(p<0.01)$ of CD34-positive capillaries in HCV compared to PSC, suggesting more angiogenesis (Figure 4C and supplementary material, Figure S5). Gene network analysis of the PSC-associated HPCs linked collagen coding genes with INHBA, reported to inhibit the growth of endothelial cells, while networks in HCV clustered around the pro-angiogenic factor IGF2 (supplementary material, Figure S6) $[27,28]$. Immunohistochemistry showed a strong protein expression of IGF2 in the endothelial cells and the surroundings of the DR in HCV compared to PSC, while INHBA was strongly positive in the endothelial cells in PSC (Figure 4D and supplementary material, Figure S2). Collectively these data show spatial and 
phenotypic differences in ECM and endothelial cells in the DR/HPCs of different chronic liver diseases.

\section{Discussion}

Ductular reaction (DR) is believed to be an expansion of the hepatic progenitor cell (HPC) compartment in an attempt of the liver to restore homeostasis and has been associated with features of progressive disease, such as portal inflammation and fibrosis [29]. In this present study we explored differences in DR in samples obtained from patients diagnosed with HCV or PSC using a high-throughput RNA sequencing approach. Our data showed that DR in the biliary diseases has a strong interaction with different extracellular matrix (ECM) markers, while the DR on a background of chronic hepatocellular damage was characterised by neo-angiogenesis. In part, our results support the hypothesis that DR is the post-natal equivalent of ductal plate formation during embryonic liver development [3]. During embryogenesis, interaction of the hepatoblast with the hepatic stellate cell precursor is crucial for the formation of intrahepatic bile ducts, while the interaction with the sinusoidal endothelial cell is pivotal for differentiation towards mature hepatocytes [30, 31]. In vitro formation of cholangiocyte-like cells also requires a strong interaction with the ECM as shown by using 3D Matrigel, whereas laminin-332 has been reported to sustain biliary features through integrin signalling $[32,33]$. This study showed that DR in HCV is associated with intense vascular remodelling at the interface hepatitis which is consistent with 
previous reports [34]. Moreover, our results showed that HCV-associated HPCs and its surrounding were enriched in metastasis-related markers, such as IGF2, NTS and PDGFRA [35-37]. The similarity with cancer progression, e.g. invasion into the parenchyma and angiogenesis, is remarkable. How the HPCs resolve ECM and induce the formation of new blood vessels in a context of chronic hepatocellular damage, and whether it is a direct or indirect effect through cross-talk with immune or endothelial cells, is still unclear. Though this indicates that even in an end-stage cirrhotic liver disease focal resolution of fibrosis can be found, which opens the discussion on how terminal the tissue scarring in cirrhotic livers is.

Although very few mature epithelial cell markers were found to be differentially expressed between the diseases, some transcriptional regulators were already focally expressed, indicating that the HPCs are at least already primed or pushed towards a certain cell fate. The hepatic nuclear factor HNF4A, which is essential for hepatocyte differentiation during mouse embryogenesis and for glycogen metabolism, was observed in periportal HPCs of HCV samples [38]. In PSC the HPCs showed a strong nuclear expression of JUN, also known as C-Jun, a protooncogene which has been associated with proliferation and oxidative stress, and has been reported to induce CXCL5 secretion in alveolar epithelium [39-41]. Interestingly, even the bile ducts showed a degree of plasticity as HNF4A and JUN were focally observed in cholangiocytes of $\mathrm{HCV}$ and PSC respectively, indicating transdifferentiation (supplementary material, Figure S2). 
In this study, several neutrophil-attractant chemokines (e.g. CXCL5, CXCL8) and oxidative stress-related genes (e.g. VNN1, NFE2L2) were found to be increased in the HPCs of PSC $[42,43]$. Infiltration of neutrophils is classically one of the features to diagnose PSC and is believed to be a response to oxidative stress. In other words, c-Jun could be a mediator induced to cope with oxidative stress in PSC and consequently regulating the transcription of neutrophil-attractant chemokines.

Besides neutrophils, PSC-associated DR/HPCs were characterised by a high infiltration of CCL28-positive macrophages. The ligand CCL28 has been reported to play an important role in attracting lymphocytes in biliary diseases [44]. Whereas in HCV samples, typical portal lymphoid follicles were observed [45]. Moreover, HCVassociated HPCs showed higher expression of MARCO, a scavenger receptor which has been implemented in bacterial clearance as part of the innate immune response [46]. In a mouse model of pulmonary fungal infection, the absence of Marco has been reported to result in a pro-inflammatory phenotype [47]. In this study, expression of MARCO was observed in the HPCs and Kupffer cells, and could reflect an anti-inflammatory state. Why HPCs express the scavenger receptor is not clear. One possibility is that MARCO expression is the result of a bacterial influx and hence reflects a hampered gut-liver signalling axis [48]. Overall, our data supports that HPCs play a crucial role in immune signalling, whether it is pro- or antiinflammatory.

In mouse models with chronic extra-hepatic biliary obstruction, the sonic hedgehog pathway has been reported to protect HPCs from apoptosis [49]. In line with these 
findings, our results showed an increase in SHH expression in the PSC-associated HPCs, together with elevated expression of the downstream transcription factor FOXJ1, which has been described to regulate cilia formation during embryogenesis [50]. Moreover, an increase in epiregulin, a mitogen of HPCs in mouse models of biliary damage, was observed in the PSC cohort [51]. This highlights the complexity in intertwined signalling cascades. DR in PSC governs growth factors to stimulate morphogenesis and protect from cholestasis, whereas DR in HCV suggests invasion of HPCs into the parenchyma. Notably, in this study we did not find any significant differences in members of the Notch pathway, a pathway pivotal for cholangiocyte differentiation [13]. Though there is a trend towards an increase in PSC, the fact that we isolated the DR/HPCs with the close surroundings rather than single HPCs could explain the lack of significance.

Although we explored the different signalling pathways in DR/HPCs, our study is limited due to its focus on end-stage liver disease and its retrospective character. The hepatocytes in end-stage PSC are often cholestatic, meaning that the comparison with HPCs should be interpreted with care. Furthermore, many of the included PSC samples have few to no remaining bile ducts, hence the alternative name vanishing bile duct disease, which translates in the lack of a proper comparison with damaged and/or normal cholangiocytes. Future work should focus on earlier stages of liver disease, not only including DR and damaged 
hepatocytes/cholangiocytes but also do a comparison with the HPC niche under healthy circumstances.

In conclusion, this present study highlighted aetiology-dependent differences in the DR/HPCs in chronic liver disease. Understanding how HPCs communicate with their surroundings might offer new therapeutic possibilities to stimulate liver regeneration and reduce inflammation, and potentially reduce fibrosis, already at an early stage of the disease. 


\section{Acknowledgements}

This work was supported by the Belgian Federal Science Policy Office, Interuniversity Attraction Poles program - P7/83-HEPRO (to OG) and the Belgian Kom op tegen Kanker (to JW).

\section{Author contributions statement}

TR, OG: study design. TR, FN, DM, WVS, MVH, OG: patient selection and clinicopathological analysis. TR, OG, MVH, KVDE: histopathology. AB: digital quantification images. OG: laser microdissection. SC, JW, WVD, RVE: RNA sequencing and bioinformatics. 


\section{References}

1. Blachier M, Leleu H, Peck-Radosavljevic M, et al. The burden of liver disease in Europe: a review of available epidemiological data. J Hepatol 2013; 58: 593-608.

2. Roskams T. Different types of liver progenitor cells and their niches. $J$ Hepatol 2006; 45: 1-4.

3. Desmet VJ. Ductal plates in hepatic ductular reactions. Hypothesis and implications. I. Types of ductular reaction reconsidered. Virchows Arch 2011; 458: $251-259$.

4. Gadd VL, Skoien R, Powell EE, et al. The portal inflammatory infiltrate and ductular reaction in human nonalcoholic fatty liver disease. Hepatology 2014; 59: 1393-1405.

5. Katoonizadeh A, Nevens F, Verslype C, et al. Liver regeneration in acute severe liver impairment: a clinicopathological correlation study. Liver Int 2006; 26: 1225-1233.

6. Sancho-Bru P, Altamirano J, Rodrigo-Torres D, et al. Liver progenitor cell markers correlate with liver damage and predict short-term mortality in patients with alcoholic hepatitis. Hepatology 2012; 55: 1931-1941.

7. Lorenzini S, Bird TG, Boulter L, et al. Characterisation of a stereotypical cellular and extracellular adult liver progenitor cell niche in rodents and diseased human liver. Gut 2010; 59: 645-654. 
8. Espanol-Suner R, Carpentier R, Van Hul N, et al. Liver progenitor cells yield functional hepatocytes in response to chronic liver injury in mice.

Gastroenterology 2012; 143: 1564-1575 e1567.

9. Peng ZW, Ikenaga N, Liu SB, et al. Integrin alphavbeta6 critically regulates hepatic progenitor cell function and promotes ductular reaction, fibrosis, and tumorigenesis. Hepatology 2016; 63: 217-232.

10. Carpino G, Renzi A, Onori $\mathrm{P}$, et al. Role of hepatic progenitor cells in nonalcoholic fatty liver disease development: cellular cross-talks and molecular networks. Int J Mol Sci 2013; 14: 20112-20130.

11. Pellicoro A, Ramachandran P, Iredale JP, et al. Liver fibrosis and repair: immune regulation of wound healing in a solid organ. Nat Rev Immunol 2014; 14: $181-194$

12. Van Hul N, Lanthier N, Espanol Suner R, et al. Kupffer cells influence parenchymal invasion and phenotypic orientation, but not the proliferation, of liver progenitor cells in a murine model of liver injury. Am J Pathol 2011; 179: $1839-1850$.

13. Boulter L, Govaere O, Bird TG, et al. Macrophage-derived Wnt opposes Notch signaling to specify hepatic progenitor cell fate in chronic liver disease. Nat Med 2012; 18: 572-579.

14. Yimlamai D, Christodoulou C, Galli GG, et al. Hippo pathway activity influences liver cell fate. Cell 2014; 157: 1324-1338. 
15. Wood MJ, Gadd VL, Powell LW, et al. Ductular reaction in hereditary hemochromatosis: the link between hepatocyte senescence and fibrosis progression. Hepatology 2014; 59: 848-857.

16. Franchitto $A$, Onori $P$, Renzi $A$, et al. Expression of vascular endothelial growth factors and their receptors by hepatic progenitor cells in human liver diseases. Hepatobiliary Surg Nutr 2013; 2: 68-77.

17. Lukacs-Kornek V, Lammert F. The progenitor cell dilemma: Cellular and functional heterogeneity in assistance or escalation of liver injury. J Hepatol 2017; 66: 619-630.

18. Trapnell C, Pachter L, Salzberg SL. TopHat: discovering splice junctions with RNA-Seq. Bioinformatics 2009; 25: 1105-1111.

19. Li H, Handsaker B, Wysoker A, et al. The Sequence Alignment/Map format and SAMtools. Bioinformatics 2009; 25: 2078-2079.

20. Anders S, Pyl PT, Huber W. HTSeq-a Python framework to work with highthroughput sequencing data. Bioinformatics 2015; 31: 166-169.

21. Morgan M, Anders S, Lawrence M, et al. ShortRead: a bioconductor package for input, quality assessment and exploration of high-throughput sequence data. Bioinformatics 2009; 25: 2607-2608.

22. Subramanian A, Tamayo P, Mootha VK, et al. Gene set enrichment analysis: a knowledge-based approach for interpreting genome-wide expression profiles. Proc Natl Acad Sci U S A 2005; 102: 15545-15550. 
23. Mootha VK, Lindgren CM, Eriksson KF, et al. PGC-1alpha-responsive genes involved in oxidative phosphorylation are coordinately downregulated in human diabetes. Nat Genet 2003; 34: 267-273.

24. Merico D, Isserlin R, Stueker O, et al. Enrichment map: a network-based method for gene-set enrichment visualization and interpretation. PLoS One 2010; 5: e13984.

25. Szklarczyk D, Morris JH, Cook H, et al. The STRING database in 2017: quality-controlled protein-protein association networks, made broadly accessible. Nucleic Acids Res 2017; 45(D1): D362-D368.

26. Walter W, Sanchez-Cabo F, Ricote M. GOplot: an R package for visually combining expression data with functional analysis. Bioinformatics 2015; 31: 2912-2914.

27. Kaneda $\mathrm{H}$, Arao T, Matsumoto K, et al. Activin A inhibits vascular endothelial cell growth and suppresses tumour angiogenesis in gastric cancer. $\mathrm{Br} \mathrm{J}$ Cancer 2011; 105: 1210-1217.

28. Chao W, D'Amore PA. IGF2: epigenetic regulation and role in development and disease. Cytokine Growth Factor Rev 2008; 19: 111-120.

29. Raven A, Lu WY, Man TY, et al. Cholangiocytes act as facultative liver stem cells during impaired hepatocyte regeneration. Nature 2017; 547: 350-354.

30. Zhang L, Theise N, Chua M, et al. The stem cell niche of human livers: symmetry between development and regeneration. Hepatology 2008; 48: 1598-1607. 
31. Sugiyama Y, Koike T, Shiojiri N. Developmental changes of cell adhesion molecule expression in the fetal mouse liver. Anat Rec (Hoboken) 2010; 293: $1698-1710$.

32. Lu WY, Bird TG, Boulter L, et al. Hepatic progenitor cells of biliary origin with liver repopulation capacity. Nat Cell Biol 2015; 17: 971-983.

33. Govaere O, Wouters J, Petz M, et al. Laminin-332 sustains chemoresistance and quiescence as part of the human hepatic cancer stem cell niche. $J$ Hepatol 2016; 64: 609-617.

34. Gabriel A, Kukla M, Wilk M, et al. Angiogenesis in chronic hepatitis $C$ is associated with inflammatory activity grade and fibrosis stage. Pathol Res Pract 2009; 205: 758-764.

35. Akter H, Park M, Kwon OS, et al. Activation of matrix metalloproteinase-9 (MMP-9) by neurotensin promotes cell invasion and migration through ERK pathway in gastric cancer. Tumour Biol 2015; 36: 6053-6062.

36. Martinez-Quetglas I, Pinyol R, Dauch D, et al. IGF2 Is up-regulated by epigenetic mechanisms in hepatocellular carcinomas and is an actionable oncogene product in experimental models. Gastroenterology 2016; 151: $1192-1205$

37. Govaere O, Petz M, Wouters J, et al. The PDGFRalpha-laminin B1-keratin 19 cascade drives tumor progression at the invasive front of human hepatocellular carcinoma. Oncogene 2017; 36: 6605-6616. 
38. Parviz F, Matullo C, Garrison WD, et al. Hepatocyte nuclear factor 4alpha controls the development of a hepatic epithelium and liver morphogenesis. Nat Genet 2003; 34: 292-296.

39. Sen CK, Packer L. Antioxidant and redox regulation of gene transcription. FASEB J 1996; 10: 709-720.

40. Seki E, Brenner DA, Karin M. A liver full of JNK: signaling in regulation of cell function and disease pathogenesis, and clinical approaches.

Gastroenterology 2012; 143: 307-320.

41. Jeyaseelan S, Manzer R, Young SK, et al. Induction of CXCL5 during inflammation in the rodent lung involves activation of alveolar epithelium. Am J Respir Cell Mol Biol 2005; 32: 531-539.

42. Nouailles G, Dorhoi A, Koch M, et al. CXCL5-secreting pulmonary epithelial cells drive destructive neutrophilic inflammation in tuberculosis. J Clin Invest 2014; 124: 1268-1282.

43. Naquet P, Pitari G, Dupre $\mathrm{S}$, et al. Role of the $\mathrm{Vnn} 1$ pantetheinase in tissue tolerance to stress. Biochem Soc Trans 2014; 42: 1094-1100.

44. Borchers AT, Shimoda S, Bowlus C, et al. Lymphocyte recruitment and homing to the liver in primary biliary cirrhosis and primary sclerosing cholangitis. Semin Immunopathol 2009; 31: 309-322.

45. Scheuer PJ, Ashrafzadeh P, Sherlock S, et al. The pathology of hepatitis C. Hepatology 1992; 15: 567-571. 
46. Arredouani MS, Palecanda A, Koziel $\mathrm{H}$, et al. MARCO is the major binding receptor for unopsonized particles and bacteria on human alveolar macrophages. J Immunol 2005; 175: 6058-6064.

47. Xu J, Flaczyk A, Neal LM, et al. Exploitation of scavenger receptor, macrophage receptor with collagenous structure, by Cryptococcus neoformans promotes alternative activation of pulmonary lymph node $\mathrm{CD} 11 \mathrm{~b}(+)$ conventional dendritic cells and non-protective Th2 bias. Front Immunol 2017; 8: 1231.

48. Tripathi A, Debelius J, Brenner DA, et al. The gut-liver axis and the intersection with the microbiome. Nat Rev Gastroenterol Hepatol 2018; 15: $397-411$.

49. Omenetti A, Popov $Y$, Jung $Y$, et al. The hedgehog pathway regulates remodelling responses to biliary obstruction in rats. Gut 2008; 57: 1275-1282.

50. Cruz C, Ribes V, Kutejova E, et al. Foxj1 regulates floor plate cilia architecture and modifies the response of cells to sonic hedgehog signalling. Development 2010; 137: 4271-4282.

51. Tomita K, Haga H, Mizuno K, et al. Epiregulin promotes the emergence and proliferation of adult liver progenitor cells. Am J Physiol Gastrointest Liver Physiol 2014; 307: G50-57. 
Table 1. Top 40 differentially expressed genes comparing DR/HPCs of HCV to PSC.

\begin{tabular}{|c|c|c|c|}
\hline Gene & Description & log2 fold-change & $P$ value \\
\hline IGHG1 & immunoglobulin heavy constant gamma 1 (G1m marker) & 3.58 & 2.11E-11 \\
\hline CYP1A1 & cytochrome P450, family 1 , subfamily A, polypeptide 1 & 6.89 & $2.83 \mathrm{E}-10$ \\
\hline IGKV3D-20 & immunoglobulin kappa variable 3D-20 & 6.56 & $5.24 \mathrm{E}-10$ \\
\hline$C D 5 L$ & CD5 molecule-like & 5.42 & $7.29 \mathrm{E}-10$ \\
\hline GPR83 & G protein-coupled receptor 83 & 6.02 & $9.26 \mathrm{E}-10$ \\
\hline$R D 3 L$ & retinal degeneration 3-like & 5.81 & 5.85E-09 \\
\hline IGHV3-43 & immunoglobulin heavy variable $3-43$ & 7.20 & 1.14E-08 \\
\hline IGFBP3 & insulin-like growth factor binding protein 3 & 2.86 & $1.78 \mathrm{E}-08$ \\
\hline GP2 & glycoprotein 2 (zymogen granule membrane) & 6.36 & 3.77E-08 \\
\hline MARCO & macrophage receptor with collagenous structure & 6.38 & 4.07E-08 \\
\hline VIPR1 & vasoactive intestinal peptide receptor 1 & 5.54 & 4.11E-08 \\
\hline IGHG3 & immunoglobulin heavy constant gamma 3 (G3m marker) & 2.84 & 7.79E-08 \\
\hline NTS & neurotensin & 4.25 & 1.30E-07 \\
\hline$R O R B$ & RAR-related orphan receptor B & 5.55 & 2.60E-07 \\
\hline IGFBP5 & insulin-like growth factor binding protein 5 & 1.70 & 3.88E-07 \\
\hline CR2 & complement component ( $3 \mathrm{~d} /$ Epstein Barr virus) receptor 2 & 6.61 & $7.22 \mathrm{E}-07$ \\
\hline IGHGP & immunoglobulin heavy constant gamma $\mathrm{P}$ (non-functional) & 2.04 & 1.03E-06 \\
\hline MS4A1 & membrane-spanning 4-domains, subfamily A, member 1 & 3.47 & 1.24E-06 \\
\hline$D A R C$ & Duffy blood group, chemokine receptor & 3.04 & 1.26E-06 \\
\hline TSPAN32 & tetraspanin 32 & 5.51 & $1.53 \mathrm{E}-06$ \\
\hline RGS4 & regulator of G-protein signaling 4 & -3.18 & $1.08 \mathrm{E}-11$ \\
\hline EREG & epiregulin & -5.62 & 7.57E-10 \\
\hline INHBA & inhibin, beta $A$ & -2.94 & 1.43E-09 \\
\hline IL8 & interleukin 8 & -3.92 & 3.27E-09 \\
\hline$R F X 4$ & regulatory factor $\mathrm{X}, 4$ & -6.64 & 3.28E-09 \\
\hline CXCL6 & chemokine ( $\mathrm{C}-\mathrm{X}-\mathrm{C}$ motif) ligand 6 & -2.78 & $1.22 \mathrm{E}-08$ \\
\hline CCL28 & chemokine (C-C motif) ligand 28 & -2.93 & $1.42 \mathrm{E}-08$ \\
\hline$A L C A M$ & activated leukocyte cell adhesion molecule & -2.03 & 1.90E-08 \\
\hline CA12 & carbonic anhydrase XII & -6.57 & 3.57E-08 \\
\hline WEE1 & WEE1 homolog (S. pombe) & -1.88 & $8.68 \mathrm{E}-08$ \\
\hline DSG2 & desmoglein 2 & -1.80 & $1.21 \mathrm{E}-07$ \\
\hline SLC23A1 & solute carrier family 23 (nucleobase transporters), member 1 & -4.92 & 1.96E-07 \\
\hline$B A R X 2$ & BARX homeobox 2 & -5.58 & 2.04E-07 \\
\hline SOX4 & SRY (sex determining region $Y$ )-box 4 & -2.59 & 2.15E-07 \\
\hline CLDN1 & claudin 1 & -1.61 & 4.88E-07 \\
\hline FOXJ1 & forkhead box J1 & -5.68 & 8.05E-07 \\
\hline VTCN1 & V-set domain containing $\mathrm{T}$ cell activation inhibitor 1 & -2.50 & 8.95E-07 \\
\hline TNFSF11 & tumor necrosis factor (ligand) superfamily, member 11 & -5.21 & $9.70 \mathrm{E}-07$ \\
\hline PLOD2 & procollagen-lysine, 2-oxoglutarate 5-dioxygenase 2 & -1.49 & 1.02E-06 \\
\hline MMP7 & matrix metallopeptidase 7 (matrilysin, uterine) & -2.71 & $1.12 \mathrm{E}-06$ \\
\hline
\end{tabular}




\section{Figure legends}

Figure 1. Isolation of the human ductular reaction (DR) / hepatic progenitor cells (HPCs) and matching parenchyma. (A) Experimental overview. Human HPCs were isolated from frozen tissue samples from patients diagnosed with $\mathrm{HCV}(n=5)$ and PSC ( $n=6)$ using laser microdissection. Matching hepatocytes were dissected from 3 patient samples per group. (B) Comparison of HPCs with hepatocytes identified 2451 differentially expressed genes in HCV and 1696 in PSC. (C, D) Gene set enrichment analysis of hepatocytes vs DR/HPCs in HCV (C) and in PSC (D).

Figure 2. Analysis of differentially expressed genes of DR/HPCs in HCV and PSC. (A) Top 10 gene ontology enrichment of the differentially expressed genes. (B) H\&E staining showing aggregates of lymphocytes typically seen in HCV, while infiltration of neutrophils is seen in PSC. IHC shows a high expression of NCAM1 in the HPCs of PSC compared to HCV ( $n=36$ ). (C) Candidate up-stream regulators. Regulatory network of HNF4A and JUN/cJun. (D) Immunohistochemical staining for Keratin 19, HNF4A and JUN in end-stage chronic liver disease $(n=36)$. Arrows indicate DR, asterisks parenchyma. (Scale bars $100 \mu \mathrm{m})$

Figure 3. Dynamics in immune signalling in DR/HPCs. (A) Differently expressed genes upregulated in HCV (red) or PSC (blue) involved in GO clusters 'immune system process', 'extracellular space' and 'cell adhesion'. (B,C) Immunohistochemical analysis and quantification for CCL28 (B) and MARCO (C) in early- $(n=20)$ and end-stage $(n=36)$ liver samples from patients diagnosed with HCV or PSC. Arrows indicate DR, asterisks Kupffer cells. Data are presented as average 
number of immunopositive cells per three high power fields, standard error of the mean, Student's $t$-test, ${ }^{*} p<0.05,{ }^{* *} p<0.01$. (Scale bars $100 \mu \mathrm{m}$ )

Figure 4. Differences in extracellular matrix deposition. (A, B) Immunohistochemical analysis for COL17A1, LAMC2, K19 and FN1 in end-stage HCV or PSC liver samples ( $n=36$ ). (C) Chromogenic double staining for K19 and CD34. CD34 positivity was analysed in areas with DR/HPCs and the entire portal tract. Data are presented as standard error of the mean, Student's t-test, ${ }^{*} p<0.05,{ }^{* *} p<0.01$. (D) Immunohistochemistry for IGF2 and INHBA in end-stage HCV or PSC liver samples. (E) Graphical abstract. (Scale bars $100 \mu \mathrm{m}$ )

\section{SUPPLEMENTARY MATERIAL ONLINE}

\section{Supplementary materials and methods NO}

Supplementary figure legends NO because legends are embedded in the Suppl Figures

Figure S1. Gene Ontology Analysis

Figure S2. Immunohistochemistry for HNF4A, JUN, CCL28, MARCO, COL17A1, LAMC2, FN1, IGF2 and INHBA 
Figure S3. Ingenuity Pathway Analysis

Figure S4. Immunohistochemistry for fibronectin 1 (FN1) and keratin 19 (K19)

Figure S5. Quantification of CD34-positive areas

Figure S6. Ingenuity Pathway Analysis.

Table S1. Clinical data discovery cohort

Table S2. Overlap up-regulated genes between the comparisons DR/hepatocytes in HCV and DR/hepatocytes in PSC

Table S3. Differentially expressed genes comparing HPCs of HCV to PSC 

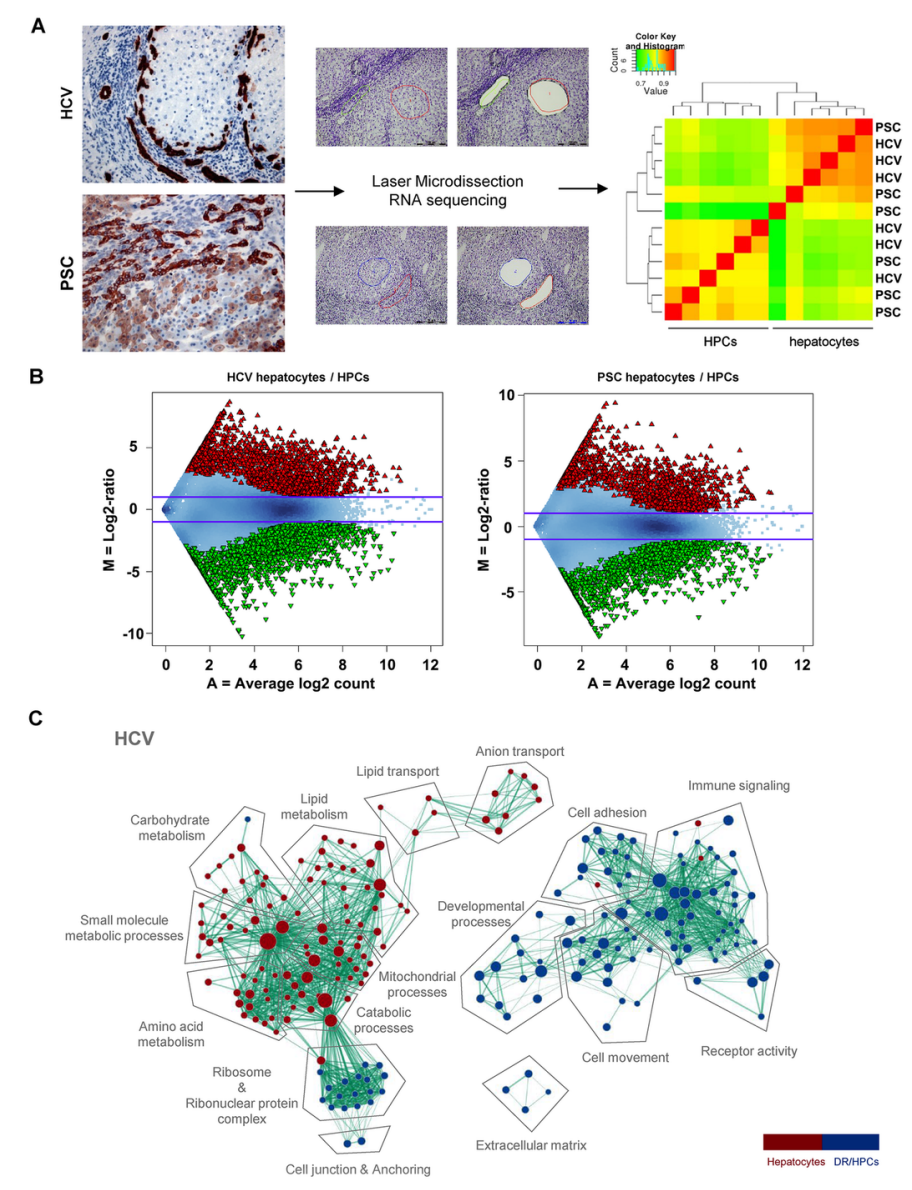

D

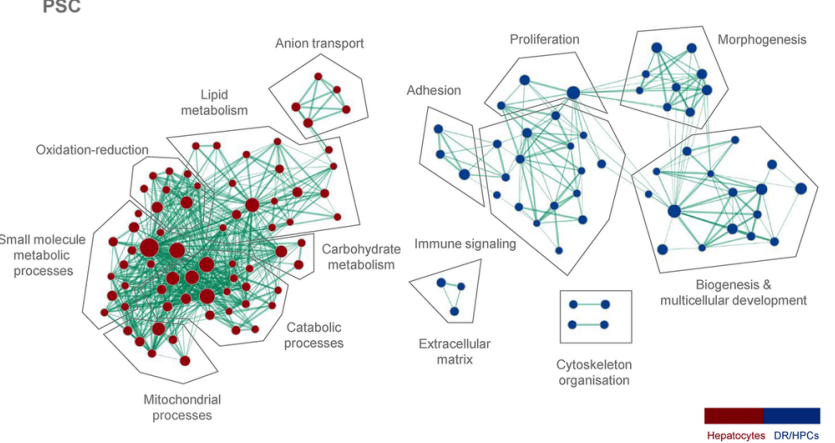

PATH_5228_Figure_1 RP.tif 

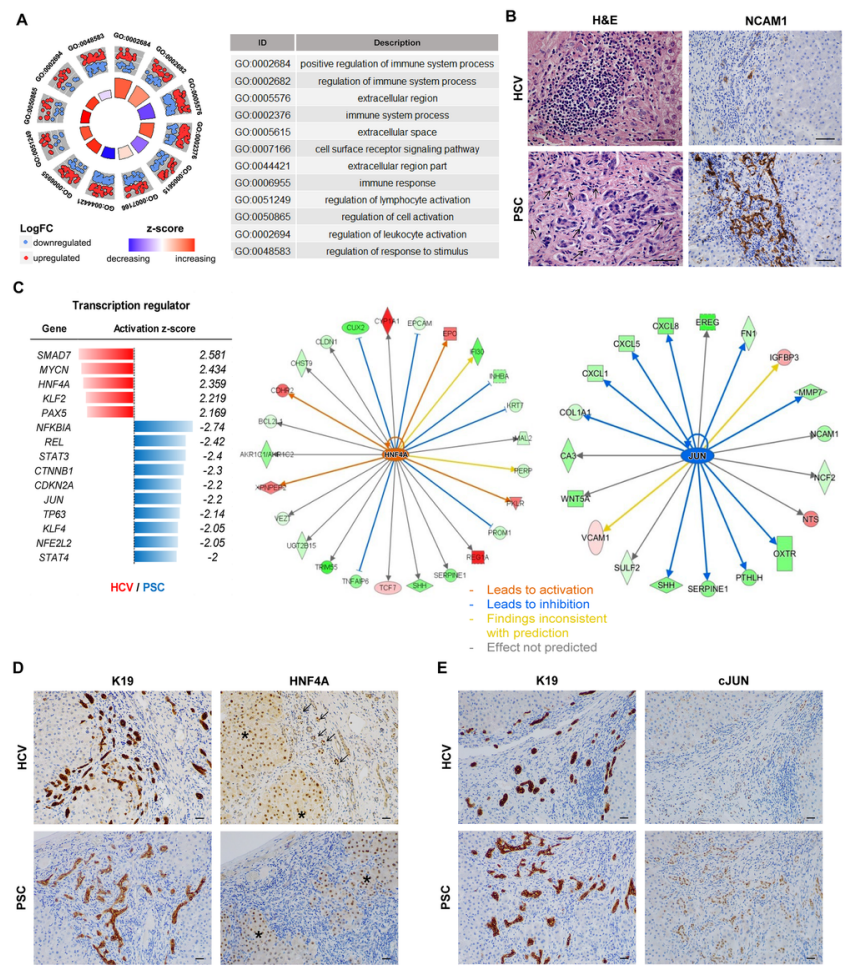

PATH_5228_Figure_2 RP.tif 


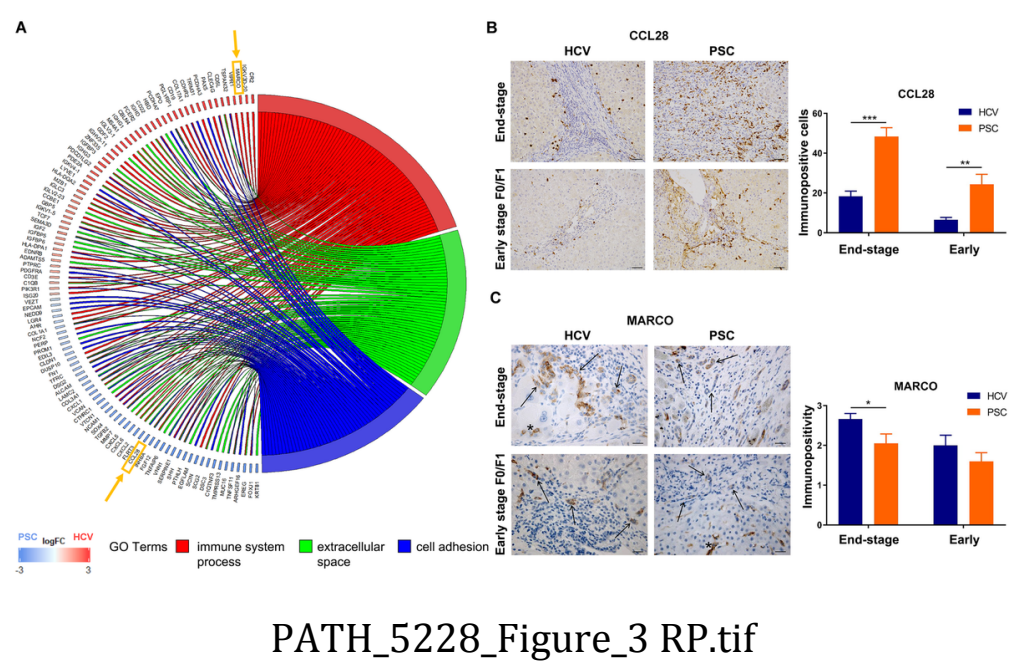

This article is protected by copyright. All rights reserved. 

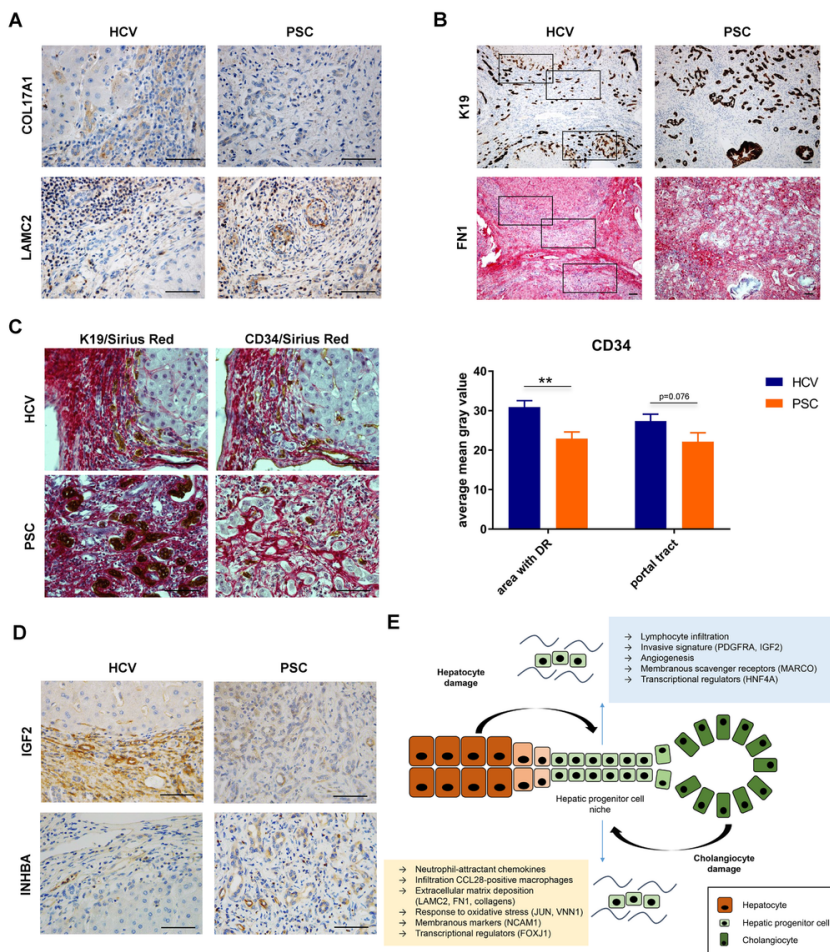

PATH_5228_Figure_4 RP.tif

This article is protected by copyright. All rights reserved. 